\title{
Stable Formation of Aqueous/Organic Parallel Two-phase Flow in Nanochannels with Partial Surface Modification
}

\author{
Hiroki SANO, ${ }^{* 1}$ Yutaka KAZOE, ${ }^{* 1, * 2 \dagger}$ and Takehiko KITAMORI $* 1, * 3, * 4 \uparrow$ \\ *1 Department of Applied Chemistry, School of Engineering, The University of Tokyo, 7-3-1 Hongo, Bunkyo, \\ Tokyo 113-8656, Japan \\ *2 Department of System Design Engineering, Faculty of Science and Technology, Keio University, 3-14-1 \\ Hiyoshi, Kohoku, Yokohama, Kanagawa 223-8522, Japan \\ *3 Collaborative Research Organization for Micro and Nano Multifunctional Devices, The University of Tokyo, \\ 7-3-1 Hongo, Bunkyo, Tokyo 113-8656, Japan \\ *4 Institute of NanoEngineering and MicroSystems, Department of Power Mechanical Engineering, National \\ Tsing Hua University, No. 101, Section 2, Kuang-Fu Road, Hsinchu 300044, Taiwan, R. O. C.
}

\begin{abstract}
In microfluidics, various chemical processes can be integrated utilizing parallel multiphase flows. Our group has extended this research to nanofluidics, and recently performed the extraction of lipids using parallel two-phase flow in nanochannels. Although this was achieved in surface-modified nanochannels, a stable condition of parallel two-phase flow remains unknown due to difficulties in device fabrication, for a suitable method of bonding surface-modified substrates is lacking. Therefore, research on parallel two-phase flow in nanochannels has been limited. Herein, a new bonding method which improves the wash process for the substrates and increases the bonding rate to $\sim 100 \%$ is described. The conditions to achieve parallel organic/aqueous two-phase flow were then studied. It was revealed that in nanochannels, higher capillary numbers for the organic phase flow were required compared to that in microchannels. The newly developed fabrication process and flow regimes will contribute to realize integrated nanofluidic devices capable of analyzing single molecules/ cells.
\end{abstract}

Keywords Nanofluidics, nanochannel, multiphase flow, bonding, surface modification, focused ion beam, femtoliter

(Received April 20, 2021; Accepted May 25, 2021; Advance Publication Released Online by J-STAGE May 28, 2021)

\section{Introduction}

In microfluidics, various chemical operations such as mixing, reaction, and extraction have been integrated to achieve micrototal analysis systems (Micro-TAS). ${ }^{1-3}$ One of the technologies widely used in such systems is multiphase flow, which consists of flows of immiscible organic/aqueous solutions., ${ }^{4,5}$ Chemical operations are miniaturized (micro unit operations (MUOs) $)^{6}$ and integrated based on this technology. Multiphase flow can be roughly categorized into two types: segmented flow and parallel flow. Segmented flow, or flows that contain droplets provide an isolated analysis field with a uniform volume. This type of flow is attractive in terms of realizing chemical reactions, cell encapsulation, analyte detection, etc., in microchannels. ${ }^{7-11}$ In addition, the efficiency of the extraction or reaction can be enhanced using droplets due to rapid mass transfer within the droplets. ${ }^{12}$ In the case of parallel multiphase flow, various chemical processes may be accomplished, as with segmented flow, ${ }^{13-17}$ but, in addition, this configuration has the advantage that MUOs can be readily integrated in the continuous flow

$\dagger$ To whom correspondence should be addressed.

E-mail: kazoe@sd.keio.ac.jp (Y. K.); kitamori@icl.t.u-tokyo.ac.jp (T. K.) system because phase confluence or phase separation can be readily achieved. Such an approach has been proposed by our group as a continuous-flow chemical process (CFCP). ${ }^{6}$ The fundamental concept here is the formation of a stable parallel multiphase flow, and various methods are needed to attain this objective. , $^{18-21}$ Given that parallel multiphase flow is maintained as a result of competing balance between the viscous force of the fluid and surface tension at the interface of the phases, control of the surface tension is an important aspect of the technology. Previously, we have fabricated a guide structure in the middle of the microchannel to form a parallel three-phase flow. ${ }^{6}$ Using the guide structure, the contact area of the different solutions involved becomes shallower and the Laplace pressure at the solution interface increases. Thus, a wider range of driving pressures is permitted in forming the stable two-phase flow. As another approach, we modified one side of the micro channel with hydrophobic molecules while the other side retained a hydrophilic glass surface. ${ }^{21}$ Such a configuration is one way to control the Laplace pressure at the interface and to stabilize the two-phase flow.

Recently, research in the microfluidics field has been downscaled to nanofluidics. ${ }^{22-27}$ Our group has been exploiting this field utilizing $10-1000 \mathrm{~nm}$ sized nanochannels on a glass substrate. ${ }^{28}$ Based on the high surface area effect in such a small volume, novel unit operations at the molecular level, 
such as single-molecule ELISA (enzyme-linked immunosorbent assay), ${ }^{29}$ single DNA molecule sorting ${ }^{30}$ nano-chromatography using the channel as a separation column, ${ }^{31}$ and picoliter enzyme reactors, ${ }^{32}$ have been performed. These operations are called nano unit operations (NUOs) and in order to interconnect NUOs and realize integrated nanofluidic analytical systems, formation of parallel multiphase flows in nanochannels is required However, in such small and confined spaces, the Laplace pressure at the interface of the two phases rises to the order of $\mathrm{MPa}$, and therefore control of the interface via the driving pressure is challenging. For this reason, partial modification of nanochannels, that is, modification of only one half side of the nanochannels, which are $100-1000 \mathrm{~nm}$ wide, is necessary. Given that the required spatial resolution is $\sim 100 \mathrm{~nm}$, conventional techniques using light and photomasks cannot be applied..$^{33,34}$ To address this difficulty, we conceived the idea of using the wall of the nanochannel as a mask and destroying the surface modification only on one half side of the nanochannel by a focused ion beam (FIB). ${ }^{35}$ With this partial modification, we succeeded in forming a parallel two-phase flow with water and dodecane in a nanochannel (1500 nm wide, $890 \mathrm{~nm}$ deep) Furthermore, using this method for formation of parallel twophase flow, integration of NUOs (phase confluence, extraction, and phase separation) was realized and successfully demonstrated for the separation of a lipid and an amino acid in a sample volume of $4 \mathrm{fL}$ (about $0.4 \%$ of the volume of a single cell) within $1 \mathrm{~ms}^{35}$

Although we succeeded to integrate NUOs utilizing a single parallel two-phase flow, the interconnection of multiple parallel two-phase flows remains a challenge, because the conditions for forming parallel two-phase flow in nanochannels are unknown. Studies by our group have revealed that water exhibits unique properties in confined spaces such as in nanochannels, for instance, higher viscosity, ${ }^{36}$ higher proton mobility, ${ }^{37}$ and lower dielectric constant. ${ }^{38}$ Therefore, the conditions for forming parallel two-phase flow in nanochannels could also be different from that in microchannels hence investigation is warranted.

Research on parallel two-phase flow in nanochannels is difficult to conduct due to the lack of a suitable method for bonding substrates with surface modifications. Usually, powerful reagents such as piranha solution (mixture of $\mathrm{H}_{2} \mathrm{SO}_{4}: \mathrm{H}_{2} \mathrm{O}_{2}=3: 1$ ) are used for the bonding of fused silica but such a pretreatment will destroy the modification as well as contamination on the surfaces. Instead, fuming nitric acid was used to both wash the surface of the substrates and maintain the modification; however, because the wash was insufficient, the success rate of the device fabrication was approximately $10 \%$. This low success rate of device fabrication represents a bottleneck for research on parallel two-phase flow in nanochannels, which is a primary reason why the conditions required for formation of stable parallel two-phase flow in nanochannels has yet to be realized.

The objective of this study is to improve the bonding process and to reveal the experimental conditions whereby sustaining stable parallel two-phase flow in nanochannels can be realized A new process for bonding glass substrates with hydrophobic modification is proposed and verified. Next, for the device fabricated with the new bonding process, the flow patterns in the nanochannels were observed. Finally, the conditions for realizing stable parallel two-phase flow in nanochannels are discussed.

\section{Channel fabrication, hydrophobic modification}

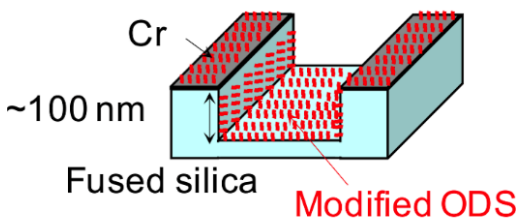

\section{FIB irradiation}

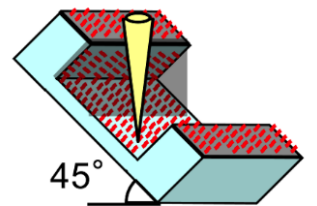

Fig. 1 Method of partial modification of the nanochannels. By tilting the substrate and using the wall of the nanochannel as a photomask, the modification on one half of the side of the nanochannel can be removed.

\section{Experimental}

\section{Fabrication process}

Microchannels and nanochannels were fabricated on glass substrates (VIO-SILSX, Shin-Etsu Quartz Co., Ltd., Tokyo, Japan) by photolithography and electron-beam lithography, followed by reactive ion etching. ${ }^{28}$ After modifying all of the nanochannels with hydrophobic molecules (octadecyltrimethoxysilane, ODS), the modification on one half of the side of the nanochannel was removed by irradiating with a FIB (Fig. 1). ${ }^{35}$ In the conventional method of bonding the substrates, the substrates are washed with a piranha solution, sonicated in water, and the surfaces are activated with an $\mathrm{O}_{2}$ plasma. However, washing with piranha solution and surface activation with the $\mathrm{O}_{2}$ plasma removes organic contaminants on the surface including the hydrophobic modification, thus the conventional bonding method cannot be applied to substrates with hydrophobic modification. To resolve this issue, a process using fuming nitric acid instead of piranha solution to wash the substrates was adopted. Sonication in water and irradiation by the $\mathrm{O}_{2}$ plasma was also eliminated from the process, for the hydrophobic modification could be destroyed by these processes.

The new fabrication process developed in this study is described in Fig. 2. First, the substrate was immersed in a chromium etchant (HICRETCH S-1, FUJIFILM Wako Pure Chemical Co.) for $8 \mathrm{~min}$ to remove the chromium. Next, before washing the substrates with fuming nitric acid, irradiation of vacuum ultraviolet (VUV) radiation was adopted to the process. Irradiation of VUV generates hydrocarbon radicals and atomic oxygen from organic compounds and molecular oxygen respectively. The fragments react with each other and eventually will be mineralized into $\mathrm{CO}_{2}$ and $\mathrm{H}_{2} \mathrm{O} .^{39}$ Thus, VUV irradiation decomposes organic components and is used as a tool for surface patterning. ${ }^{29}$ In this study, VUV was irradiated for $12 \mathrm{~min}$ in order to clean the surface of the substrates. Then, the substrates were washed with fuming nitric acid for $8 \mathrm{~min}$, and then sonicated in water for $10 \mathrm{~min}$. The other substrate was washed using the conventional process (washing with piranha solution, sonication, and surface activation with the $\mathrm{O}_{2}$ plasma). Finally, the two substrates were bonded. 
1. Cr etching

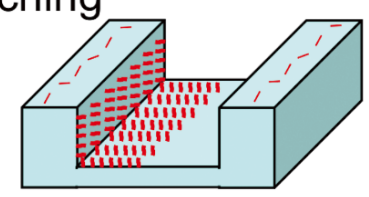

2. VUV exposure

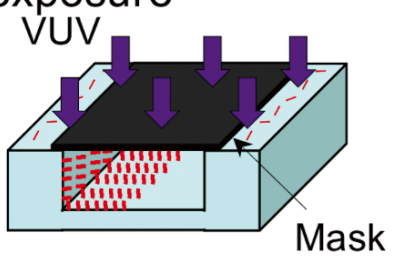

3. Substrate washing (Fuming $\mathrm{HNO}_{3}$, Sonication)

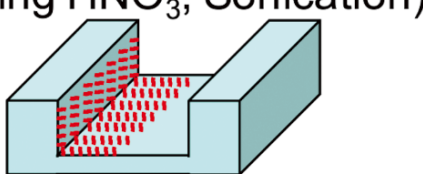

\section{Low temperature bonding}

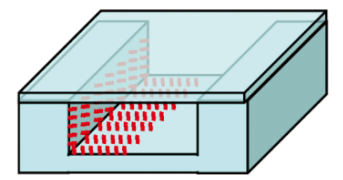

Fig. 2 Fabrication process. After removal of chromium by the etching solution, the substrates are irradiated with VUV radiation to remove organic contaminants on the substrate surfaces. Next, the substrates are washed with fuming nitric acid and then sonicated in ultrapure water.

Table 1 The washing process proposed in this study and the washing process in previous study

\begin{tabular}{llll} 
& & Previous study & \multicolumn{1}{c}{ This study } \\
\hline Step 1 & Modification & ODS & ODS \\
Step 2 & Cr etching time & $3-4$ min & Longer time \\
Step 3 & VUV irradiation & - & 12 min \\
Step 4 & Washing & Fuming nitric acid & Fuming nitric acid \\
Step 5 & Sonication & - & 10 min \\
Step 6 & Bonding & Low temperature & $\begin{array}{c}\text { Low temperature } \\
\text { bonding }\end{array}$ \\
& & bonding & bong
\end{tabular}

The conventional process and the new process adopted in this study are compared in Table 1. In a typical chromium lift-off process, the time for etching is around $1 \mathrm{~h}$, but, in this case, using an extended immersion time for the chromium etchant would affect the hydrophobicity. In the conventional procedure, the chromium etching time was only 3 to $4 \mathrm{~min}$, and this was just the length that $\mathrm{Cr}$ on the surface was apparently removed. However, if the etching time is too short, chromium could remain on the surface of the substrate, which makes bonding impossible. Therefore, in this study, we investigated the relationship between the etching time and the hydrophobicity of ODS and a process with a longer etching time was used. In addition, a new wash process before bonding of the substrates was also included. In the conventional bonding process, the substrates are washed with piranha solution, sonicated in water, and the surface is activated by the $\mathrm{O}_{2}$ plasma. In this study, $12 \mathrm{~min}$ of VUV irradiation with a photomask covering the modified area was applied before washing with fuming nitric acid, which was then followed by $10 \mathrm{~min}$ of sonication in water. After these pretreatments the two substrates were bonded using a low temperature bonding procedure. ${ }^{40}$

\section{Verification of hydrophobicity}

To verify the hydrophobicity of the nanochannel after performing the new fabrication process, the contact angle of water was measured by a DropMaster DM500 unit (Kyowa Interface Science Co., Ltd.). By undertaking measurements successively after immersion in the chromium etchant, VUV exposure, and sonication in water, the hydrophobicity was verified.

Device fabrication and formation of parallel two-phase flow

Two devices with different channel sizes at the phase confluence were fabricated: one device was $3.8 \mu \mathrm{m}$ wide and $1.8 \mu \mathrm{m}$ deep, and the other was $1000 \mathrm{~nm}$ wide and $470 \mathrm{~nm}$ deep. The length of the channel after the confluent point in both devices was $30 \mu \mathrm{m}$. As a general-purpose extraction solvent, chloroform served as the organic phase and ultrapure water was used as the aqueous phase. The reagents were flowed in from different microchannels with a width of $500 \mu \mathrm{m}$ and a depth of $6 \mu \mathrm{m}$ connected to the nanochannels using a pressure controller. The two streams were merged at a Y-shaped junction. After the confluence point, the two solutions flow through a $30 \mu \mathrm{m}$-long confluent channel and separate at the exit. The pressure of the aqueous phase was varied with an interval of $5 \mathrm{kPa}$ while the pressure of the organic phase was fixed at $0,25,50,75,100$, $125,150,175$, and $200 \mathrm{kPa}$, respectively. The flow patterns were observed with an inverted microscope (IX71, Olympus Corp., Tokyo, Japan) combined with an oil immersion objective lens $(100 \mathrm{X}, \mathrm{NA}=1.4)$ and an electron multiplying chargecoupled device (EMCCD) camera (C9100-13, Hamamatsu Photonics K. K., Hamamatsu, Japan), and the conditions for forming parallel two-phase flow were studied.

\section{Results and Discussion}

\section{Verification of hydrophobicity}

To verify the hydrophobicity by measurement of the contact angle, the surface of the substrate was modified with ODS in the same way as the nanochannels were modified. The contact angle just after the modification with ODS was $95.4^{\circ}$. The relationship between the contact angle and the immersion time in the etchant is shown in Fig. 3(a). Although the hydrophobicity gradually decreased as the immersion time increased, the surface was still hydrophobic after 8 min of immersion. However, after immersion of the substrate in the etchant for $10 \mathrm{~min}$, the contact angle decreased to $85^{\circ}\left(<90^{\circ}\right)$, which indicates that the surface became hydrophilic. The chromium etchant is an oxidant consisting of ammonium cerium(IV) nitrate; thus, it is probable that immersion in the etchant results in decomposition of the carbon chain of ODS, and therefore the hydrophobicity is reduced. 
(a)

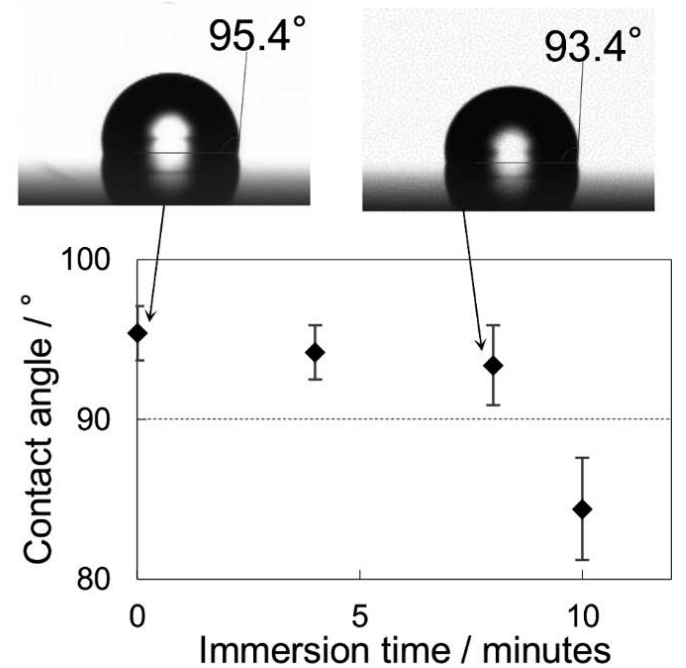

(b)

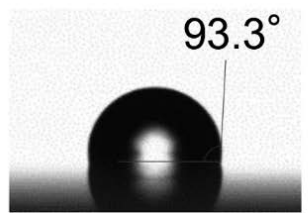

(c)

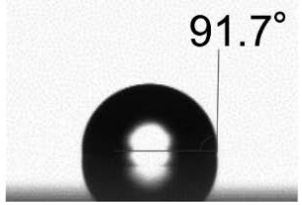

Fig. 3 Contact angle measurements on the surface of the modified substrate. (a) Measurement just after ODS modification and measurement after immersion of the substrate in etchant for 4, 8, and 10 min. (b) Measurement after VUV irradiation with a photomask. (c) Measurement after VUV irradiation and 10-min of sonication.

Next, using a mask to protect the modified area, the substrate, which had been immersed in etchant for $8 \mathrm{~min}$, was irradiated with VUV radiation and the contact angle was measured. After the measurement, the substrate was sonicated in pure water for $10 \mathrm{~min}$ and the contact angle was again measured. Images of the measurements are presented in Figs. 3(b) and 3(c). Both results suggest that the hydrophobicity was maintained after VUV irradiation and sonication. Based on these results, the etching time was lengthened to $8 \mathrm{~min}$, and the steps involving the VUV irradiation with a photomask covering the modified area and sonication were incorporated into the bonding protocol.

\section{Device fabrication}

The results for the new bonding process are depicted in Fig. 4. With the previous process, there were many voids or unbonded areas remaining on the device (Fig. 4(a)), but with the new process, no voids were apparent on the device (Fig. 4(b)). The bonding success rate was nearly $100 \%$ and a clear improvement in bonding was achieved. Using the new bonding process, two devices were fabricated as shown in Fig. 5. Two different sized Y-shaped nanochannels, that is, of microscale and nanoscale dimensions, were fabricated and investigated.

\section{Formation of parallel two-phase flow}

The flow results are shown in Fig. 6. For both devices, three patterns of flow were observed as the driving pressure was changed: single-phase flow of aqueous phase, single-phase flow

(a)

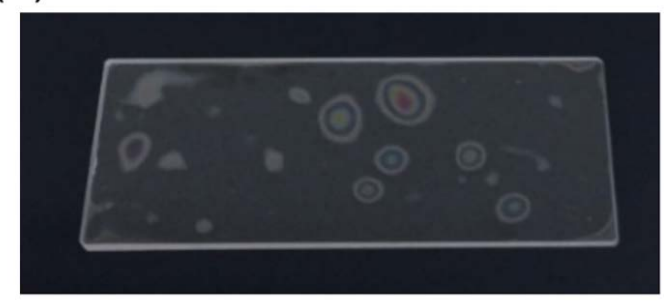

(b)

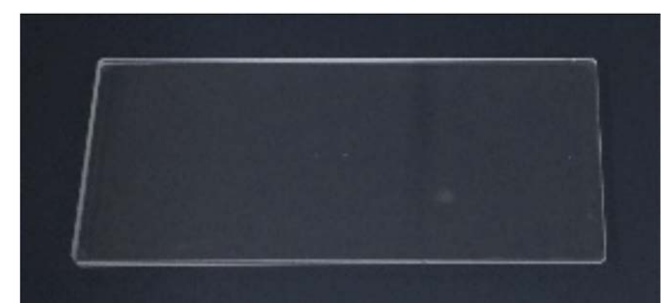

Fig. 4 Results for bonding with different wash processes. Conventional wash process. (b) New wash process. (a)

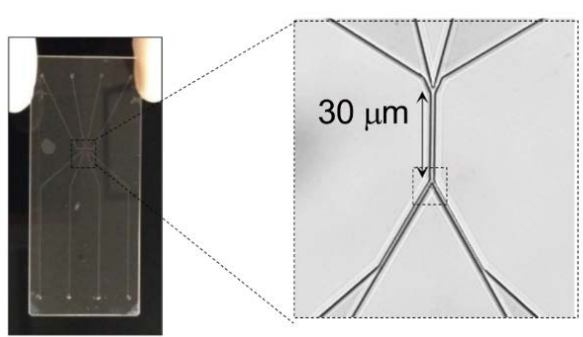

(b)
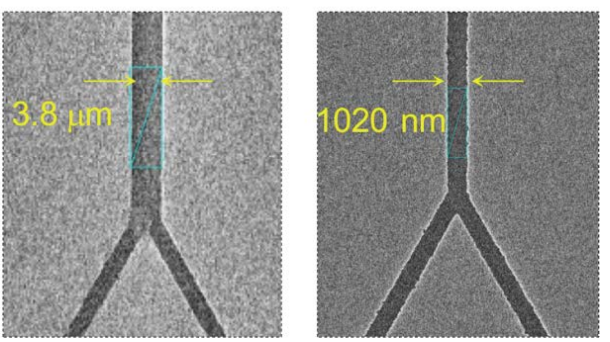

Fig. 5 (a) Schematic and a microscopy image of the fabricated device. (b) SEM image of the nanochannel on each device: a $3.8 \mu \mathrm{m}$ wide microchannel (left), and a $1020 \mathrm{~nm}$ wide nanochannel (right).

of organic phase, and parallel two-phase flow. The flow pattern was determined as being parallel two-phase flow when the two solutions were clearly separated at the exit of the nanochannel. Because parallel two-phase flow in the nanochannel was not formed when both the pressure of organic and aqueous phase were $0 \mathrm{kPa}$, the lowest pressure of the organic phase in order to form parallel two-phase flow was experimentally obtained, and additionally plotted in Fig. 6(b). The pressure at this point was $3 \mathrm{kPa}$ for the organic phase and $0 \mathrm{kPa}$ for the aqueous phase.

The condition for the formation of parallel two-phase flow is discussed using the capillary number $\mathrm{Ca}$, which is the ratio of 
(a)

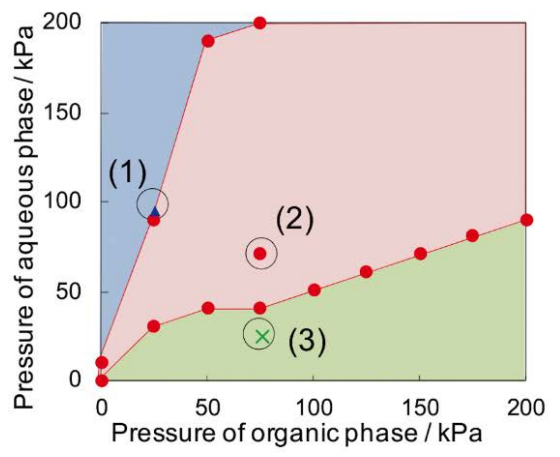

(1)

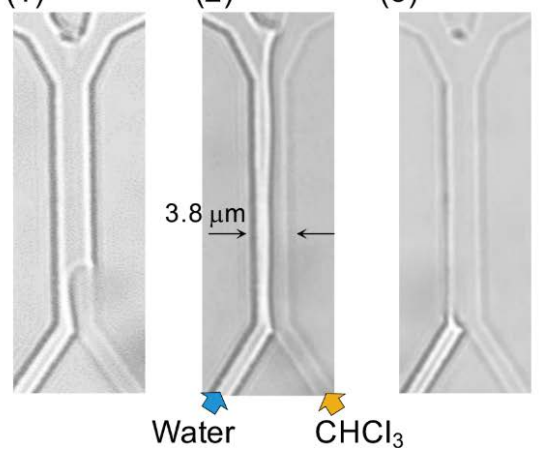

(b)

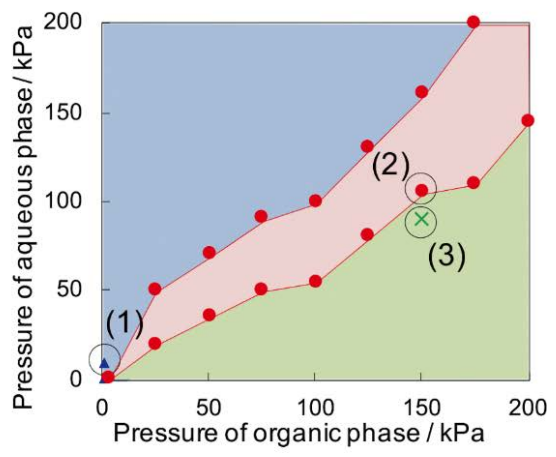

(1)

(2)

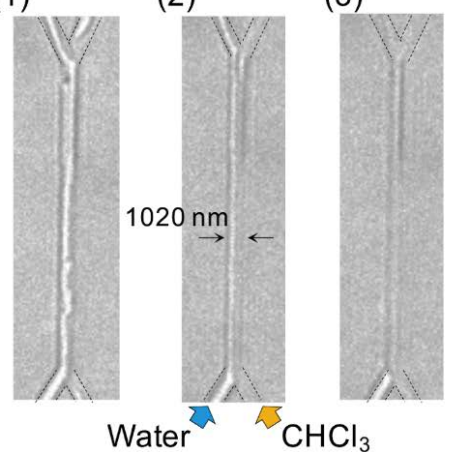

Fig. 6 Relationship between the balance of the driving pressure of water and chloroform and the flow patterns. The red circles, blue triangles, and green cross marks represent the pressure condition for forming parallel two-phase flow, single aqueous phase flow, and single organic phase flow, respectively. The pink, blue, green shaded areas are areas where parallel two-phase flow, single water phase flow, single organic phase flow was observed respectively. (a) Results of the experiments performed in the microchannels. (b) Results of the experiments performed in the nanochannels.

the viscous force to the surface tension. The capillary number may be used to discuss whether a flow will form a parallel flow or generates droplets, ${ }^{41,42}$ and is expressed as

$$
C a=\frac{\mu V}{\sigma},
$$

where $\mu, V$, and $\sigma$ are the viscosity, velocity, and the interfacial tension of the solution, respectively. Given that the flow in a nanochannel can be considered to be laminar flow, the HagenPoiseuille law can be applied and, therefore, the velocity may be estimated as

$$
V=\frac{D^{2}}{32 \mu L} \Delta P
$$

where $\Delta P$ is the pressure drop through the nanochannel, $D$ is the equivalent diameter of the nanochannel, and $L$ is the length of the nanochannel. From the two equations above, the pressure can be transformed into capillary number. When a parallel twophase flow is formed in microchannels, the conditions for the capillary numbers can be expressed as $0.46<C a_{\mathrm{aq}} / C a_{\mathrm{org}}<3.8$. However, the condition in the nanochannels was $0.63<$ $C a_{\text {aq }} / C a_{\text {org }}<1.1$, where $C a_{\text {org }}$ and $C a_{\text {aq }}$ are the capillary numbers of the organic phase and the aqueous phase, respectively. Given that the conditions in the microchannels for this study were in good agreement with previous reports on parallel two-phase flow in microchannels, ${ }^{43,44}$ this result implies that the ratio of the capillary numbers $\left(\mathrm{Ca}_{\mathrm{aq}} / \mathrm{Ca}_{\mathrm{org}}\right)$ required to form parallel two- phase flow is, on average, lower in nanochannels compared to that in microchannels. From a previous report, it is known that the viscosity of water rises in confined spaces such as nanochannels, while there are no property changes for organic solutions. ${ }^{36}$ It is also known that interfacial tension does not change in nanochannels. ${ }^{36}$ Considering these facts, the higher viscosity of water in the nanochannels could increase the viscous force of the water flow. In order to maintain a parallel two-phase flow, a higher capillary number for the organic phase would be required, which could be a possible reason for why a lower ratio of capillary numbers $\left(C a_{\mathrm{aq}} / C a_{\text {org }}\right)$ are required to form parallel two-phase flow.

The conditions obtained in this work to form parallel twophase flow in nanochannels should serve as guidelines to aid design and realize integrated analytical devices that consist of interconnected parallel multiphase flows. This development will contribute to the realization of nanofluidic devices capable of analyzing single molecules/cells.

\section{Conclusions}

In this study, the bonding process of substrates with hydrophobic modifications has been improved, and therefore we have been able to reveal robust conditions for formation of stable parallel two-phase flow in nanochannels. For the new bonding process, the etching time of chromium was extended to reduce the residual chromium on the surface. In addition, the surface was irradiated with VUV radiation to remove organic contaminants 
before washing with fuming nitric acid, and the substrate was sonicated in ultrapure water to remove particles on the surface. The new process was verified, and the success rate of the bonding rose to nearly $100 \%$. Next, two devices with microscale and nano-scale confluent channels, respectively, were fabricated with the new process and the flow patterns in the nanochannels were observed. Three types of flow were observed depending on the driving pressure of the organic and aqueous solutions. Finally, the conditions for forming stable parallel two-phase flow were discussed using capillary number theory. For flow in the nanochannel, relatively larger capillary numbers for the organic phase were required in order to form stable parallel two-phase flow compared to that in the microchannel. The increase in the viscosity of water in the nanochannels could be a possible cause for this difference. Using this novel bonding technology, conditions for achieving stable parallel two-phase flow in nanochannels have been revealed, and this would contribute to realizing integrated nanofluidic devices that are capable of processing single cells or even sample volumes smaller than a single cell.

\section{Acknowledgements}

The authors gratefully acknowledge the financial support of the Core Research for Evolutional Science and Technology (CREST) of the Japan Science and Technology Agency (JST): JPMJCR14G1. Fabrication and observation facilities were provided in part by the Academic Consortium for Nano and Micro Fabrication of four universities (The University of Tokyo, Tokyo Institute of Technology, Keio University, and Waseda University, Japan).

\section{References}

1. A. Manz and H. Becker, "Microsystem Technology in Chemistry and Life Science", 1998, Springer-Verlag Berlin Heidelberg, Berlin.

2. A. Günther and K. F. Jensen, Lab Chip, 2006, 6, 1487.

3. Y. C. Chung, Y. C. Lin, M. Z. Shiu, and W. N. T. Chang, Lab Chip, 2003, 3, 228.

4. J. D. Tice, H. Song, A. D. Lyon, and R. F. Ismagilov, Langmuir, 2003, 19, 9127.

5. K. Mawatari, Y. Kazoe, A. Aota, T. Tsukahara, K. Sato, and T. Kitamori, J. Flow Chem., 2011, 1, 3.

6. M. Tokeshi, T. Minagawa, K. Uchiyama, A. Hibara, K. Sato, H. Hisamoto, and T. Kitamori, Anal. Chem., 2002, 74, 1565.

7. H. Song, D. L. Chen, and R. F. Ismagilov, Angew. Chem. Int. Edit., 2006, 45, 7336.

8. P. Mary, V. Studer, and P. Tabeling, Anal. Chem., 2008, 80, 2680.

9. H. Shen and Q. Fang, Talanta, 2008, 77, 269.

10. M. C. Morales and J. D. Zahn, Microfluid. Nanofluid., 2010, 9, 1041.

11. J. F. Edd, D. D. Carlo, K. J. Humphry, S. Köster, D. Irimia, D. A. Weitz, and M. Toner, Lab Chip, 2008, 8, 1262.

12. J. H. Xu, J. Tan, S. W. Li, and G. S. Luo, Chem. Eng. J., 2008, 141, 242.

13. G. Münchow, S. Hardt, J. P. Kutter, and K. S. Drese, Lab Chip, 2007, 7, 98.
14. D. Ciceri, J. M. Perera, and G. W. Stevens, Microfluid. Nanofluid., 2011, 11, 593.

15. U. Novak, A. Pohar, I. Plazl, and P. Znidarsic-Plazl, Sep. Purif. Technol., 2012, 97, 172.

16. P. Znidarsic-Plazl and I. Plazl, Lab Chip, 2007, 7, 883.

17. M. D. R. Payán, H. Jensen, N. J. Petersen, S. H. Hansen, and S. Pedersen-Bjergaard, Anal. Chim. Acta, 2012, 735, 46.

18. L. R. Mason, D. Ciceri, D. J. E. Harvie, J. M. Perera, and G. W. Stevens, Microfluid. Nanofluid., 2013, 14, 197.

19. B. Yamawaki, R. Mori, K. Tsukagoshi, K. Tsuchiya, K. Yamashita, and M. Murata, Anal. Sci., 2019, 35, 249.

20. V. Reddy and J. D. Zahn, J. Colloid Interf. Sci., 2005, 286, 158.

21. A. Aota, A. Hibara, and T. Kitamori, Anal. Chem., 2007, 79, 3919.

22. D. Xia, J. Yan, and S. Hou, Small, 2012, 8, 2787.

23. V. Müller and F. Westerlund, Lab Chip, 2017, 17, 579.

24. P. Abgrall and N. T. Nguyen, Anal. Chem., 2008, 80, 2326.

25. H. Babar and H. M. Ali, J. Mol. Liq., 2019, 281, 598.

26. A. Arshad, M. Jabbal, Y. Yan, and D. Reay, J. Mol. Liq., 2019, 279, 444.

27. J. M. Munyalo and X. Zhang, J. Mol. Liq., 2018, 265, 77.

28. K. Morikawa, Y. Kazoe, Y. Takagi, Y. Tsuyama, Y. Pihosh, T. Tsukahara, and T. Kitamori, Micromachines-Basel, 2020, 11, 995.

29. K. Shirai, K. Mawatari, and T. Kitamori, Small, 2014, 10, 1514.

30. B. R. Cipriany, P. J. Murphy, J. A. Hagarman, A. Cerf, D.Latulippe, S. L. Levy, J. J. Benítez, C. P. Tan, J. Topolancik,P. D. Soloway, and H. G. Craighead, Proceedings of the National Academy of Sciences of the United Stated of America, 2012, 109, 8477.

31. R. Ishibashi, K. Mawatari, and T. Kitamori, Small, 2012, 8 , 1237.

32. K. Yamamoto, K. Morikawa, H. Imanaka, K. Imamura, and T. Kitamori, Analyst, 2020, 145, 5801.

33. B. Zhao, J. S. Moore, and D. J. Beebe, Science, 2001, 291, 1023.

34. M. B. Romanowsky, M. Heymann, A. R. Abate, A. T. Krummel, S. Fraden, and D. A. Weitz, Lab Chip, 2010, 10, 1521.

35. Y. Kazoe, T. Ugajin, R. Ohta, K. Mawatari, and T. Kitamori, Lab Chip, 2019, 19, 3844.

36. L. Li, Y. Kazoe, K. Mawatari, Y. Sugii, and T. Kitamori, J. Phys. Chem. Lett., 2012, 3, 2447.

37. T. Tsukahara, A. Hibara, Y. Ikeda, and T. Kitamori, Angew. Chem. Int. Edit., 2007, 46, 1180.

38. K. Morikawa, Y. Kazoe, K. Mawatari, and T. Kitamori, Anal. Chem., 2015, 87, 1475.

39. Z. Falkenstein, Proceedings of the Society of Photo-optical Instrumentation Engineers (SPIE), 2001, 4440, 246.

40. R. Ohta, K. Mawatari, T. Takeuchi, K. Morikawa, and T. Kitamori, Biomicrofluidics, 2019, 13, 024104

41. J.D. Tice, A. D. Lyon, and R. F. Ismagilov, Anal. Chim. Acta, 2004, 507, 73 .

42. J. D. Tice, H. Song, A. D. Lyon, and R. F. Ismagilov, Langmuir, 2003, 19, 9127.

43. A. Hibara, M. Tokeshi, K. Uchiyama, H. Hisamoto, and T. Kitamori, Anal. Sci., 2001, 17, 89.

44. A. Aota, K. Mawatari, and T. Kitamori, Lab Chip, 2009, 9 , 2470 . 